\title{
ERFAHRUNGEN
}

\section{Geld oder Liebe}

\section{Joachim Speicher}

\begin{abstract}
Bereits vor fast Jahren begann in Rheinland-Pfalz die Erprobung Persönlicher Budgets in der Behindertenhilfe. Alternativ zur üblichen Sachleistung wurden pauschalierte Geldbeträge angeboten (»Geld oder Liebe? «). Ein Blick auf die bisherigen Erfahrungen zeigt, dass viele behinderte Menschen und ihre Angehörigen diese neue Option ebenso nutzen wie die Leistungsträger und manche Leistungserbringer.
\end{abstract}

Im Jahr 1997 führte Florian Gerster als Sozialminister in Rheinland-Pfalz Persönliche Budgets in der Behindertenhilfe ohne die sonst übliche Abstimmung mit den Wohlfahrtsverbänden ein. Die »Geldleistung « als Alternative zur »Sachleistung « wurde trotz vielfacher Proteste über diesen Akt der »Vollstreckung « installiert. (1)

Noch heute, im Jahr 2006, werden die seinerzeit durch das Ministerium einseitig festgesetzten Rahmenbedingungen und Teilnahmevoraussetzungen in Rheinland-Pfalz angewendet, obwohl seit dem 1. Juli 2004 bundesweit das trägerübergreifende Persönliche Budget nach § 17 SGB IX (vgl. Kasten Seite 11) eingeführt worden ist. Ermöglicht wird diese Situation durch den neuen Absatz 5 im $§ 17$ SGB IX. Alle Modellerprobungen zum Persönlichen Budget, die vor dem 1. Juli 2004 in den Bundesländern begonnen wurden, dürfen in der am 30. Juni 2004 geltenden alten Fassung des $\S 17$ Absatz 3 SGB IX bis zum 31. Dezember 2007 fortgeführt werden. (2)

In nahezu allen 36 rheinland-pfälzischen kommunalen Gebietskörperschaften ist das Verfahren etabliert. Wohl hat sich aber eine Umsetzungspraxis entwickelt, die höchst heterogen von jenen Vorgaben aus dem Jahr 1997 abweicht — im Positiven wie im Negativen. Die Heterogenität (manchmal geht auch das Wort der »Beliebigkeit « um) betrifft die Fragen des Zugangsrechts zum Persönlichen Budget genauso wie die Regelungen zum Antragsverfahren und der Bedarfsfeststellung sowie das geänderte Rechtsverhältnis zwischen Leistungsträger, Leistungserbringer und Hilfeberechtigtem.

Ende 2003 hatte der Bundesgesetzgeber im »Gesetz zur Einordnung des Sozialhilferechts in das Sozialgesetzbuch « eine neue Ausgangslage geschaffen, die eine deutliche Verbesserung der bisherigen rheinland-pfälzischen Lösungen sowie darüber hinaus eine bundesweite Installierung des Persönlichen Budgets zur Folge hatte.

Wenn wir über das Thema »Persönliches Budget« sprechen, so zeigt sich durchaus eine gewisse begriffliche Konfusion. Konkret gibt es in Deutschland vier, sich wesentlich von einander unterscheidende Budgettypen:

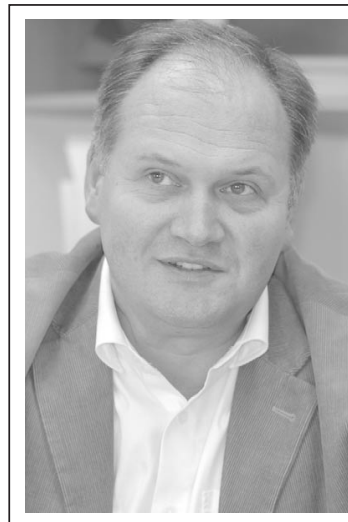

Joachim Speicher (45) ist Diplompädagoge und stellvertretender Landesgeschäftsführer des Paritätischen Wohlfahrtsverbandes, Landesverband RheinlandPfalz/Saarland. Er ist Gesamtleiter des Paritätischen Gesundheitszentrums Mainz.

\section{E-Mail Joachim.Speicher@paritaet.org}

- das Persönliche Budget in Rheinland-Pfalz (BSHG/SGB XII) seit 1998 (3)

- das trägerübergreifende Persönliche Budget (SGB IX) seit 2004 (4)

- das Pflegebudget (SGB XI) seit 2004 (5)

- das Integrierte Budget in Rheinland-Pfalz (eine Kombination aus dem trägerübergreifendem Persönlichen Budget und dem Pflegebudget) seit 2005 (6)

Eine umfassende Zahl an Praxisbeispielen einer alltäglichen Anwendungspraxis Persönlicher Budgets gibt es allerdings bisher nur in Rheinland-Pfalz zum ersten Budgettyp. Seit der Ankündigung und Einführung im Jahr 1997 ist die Zahl der Budgetnehmerinnen und Budgetnehmer auf aktuell 1.400 gestiegen und sie nimmt stetig zu. Den größten Anteil mit knapp der Hälfte stellen Menschen mit seelischer Behinderung; gefolgt von Menschen mit geistiger Behinderung oder Lernschwierigkeiten sowie Menschen mit Körper- und Sinnesbehinderungen.

Die Besonderheit rheinland-pfälzischer Budgets besteht darin, dass sich die Verfahren und die sonstigen Rahmenbedingungen mit den jetzt gültigen, in der Budgetverordnung (nach § 21a SGB IX) festgeschriebenen Regeln kaum vereinbaren lassen. (7) Auf den Punkt gebracht: Rheinland-pfälzische Persönliche Budgets sind absolut vergleichbar und in der Höhe sogar identisch mit der als »Pflegegeld « ausgezahlten Geldleistung in der Pflegeversicherung. Daher sind sie im Unterschied zu den in der Erprobung befindlichen trägerübergreifenden Persönlichen Budgets weder bedarfsorientiert, noch bedarfsdeckend und auch nicht zielorientiert. Es handelt sich vielmehr um pauschalierte Geldbeträge, die dem Menschen mit Behinderung monatlich bar auf sein Girokonto überwiesen werden und die er zur Sicherung seiner Teilhabe am Leben in der Gesellschaft frei und ohne Nachweispflicht verwenden kann. 


\section{Ausführung von Leistungen, Persönliches Budget}

(1) Der zuständige Rehabilitationsträger kann Leistungen zur Teilhabe

1. allein oder gemeinsam mit anderen Leistungsträgern

2. durch andere Leistungsträger oder

3. unter Inanspruchnahme von geeigneten, insbesondere auch freien und gemeinnützigen oder privaten Rehabilitationsdiensten und -einrichtungen (§ 19)

ausführen. Er bleibt für die Ausführung der Leistungen verantwortlich. Satz 1 gilt insbesondere dann, wenn der Rehabilitationsträger die Leistung dadurch wirksamer oder wirtschaftlicher erbringen kann.

(2) Auf Antrag können Leistungen zur Teilhabe auch durch ein monatliches Persönliches Budget ausgeführt werden, um den Leistungsberechtigten in eigener Verantwortung ein möglichst selbstbestimmtes Leben zu ermöglichen. Bei der Ausführung des Persönlichen Budgets sind nach Maßgabe des individuell festgestellten Bedarfs die Rehabilitationsträger, die Pflegekassen und die Integrationsämter beteiligt. Das Persönliche Budget wird von den beteiligten Leistungsträgern trägerübergreifend als Komplexleistung erbracht. Budgetfähige Leistungen sind Leistungen, die sich auf alltägliche, regelmäßig wiederkehrende und regiefähige Bedarfe beziehen und als Geldleistungen oder durch Gutscheine erbracht werden können. Eine Pauschalierung weiterer Leistungen bleibt unberührt. An die Entscheidung ist der Antragsteller für die Dauer von sechs Monaten gebunden.

(3) Persönliche Budgets werden in der Regel als Geldleistung ausgeführt. In begründeten Fällen sind Gutscheine auszugeben. Persönliche Budgets werden im Verfahren nach § 10 so bemessen, dass der individuell festgestellte Bedarf gedeckt wird und die erforderliche Beratung und Unterstützung erfolgen kann. Dabei soll die Höhe des Persönlichen Budgets die Kosten aller bisher individuell festgestellten, ohne das Persönliche Budget zu erbringenden Leistungen nicht überschreiten.

(4) Enthält das Persönliche Budget Leistungen mehrerer Leistungsträger, erlässt der nach § 14 erstangegangene und beteiligte Leistungsträger im Auftrag und im Namen der anderen beteiligten Leistungsträger den Verwaltungsakt und führt das weitere Verfahren durch.

(5) § 17 Abs. 3 in der am 30. Juni 2004 geltenden Fassung findet auf Modellvorhaben zur Erprobung der Einführung Persönlicher Budgets weiter Anwendung, die vor In-Kraft-Treten dieses Gesetzes begonnen haben.

(6) In der Zeit vom 1. Juli 2004 bis zum 31. Dezember 2007 werden Persönliche Budgets erprobt. Dabei sollen insbesondere modellhaft Verfahren zur Bemessung von budgetfähigen Leistungen in Geld und die Weiterentwicklung von Versorgungsstrukturen unter wissenschaftlicher Begleitung und Auswertung erprobt werden.

\section{$\S 17$ SGB IX}

Sie werden zusätzlich zu einer laufenden Grundsicherung oder Hilfe zum Lebensunterhalt gezahlt.
Diese Besonderheit hat ein rheinland-pfälzischer Psychiatrie-Erfahrener kürzlich sehr treffend auf den Punkt gebracht, als er beiläufig erwähnte, dass er selbst schon mehr als zwei Jahre lang »Schizophreniegeld « (in einer treffenden Analogie zum »Blindengeld «) beziehe.

Nun liegt die Frage nahe, auf welche Weise die Budgetinhaber ihre Teilhabe herstellen und wie sie diese sichern, wenn die Geldbeträge doch pauschaliert und im Unterschied zu den Sachleistungskosten beispielsweise einer Wohnheimunterbringung äußerst knapp bemessen sind. (8) Hierzu im Folgenden vier Praxisbeispiele und Fallvignetten.

\section{Beispiel 1: Fitness-Studio statt Tagesförderstätte}

Ein junger Mann (24 Jahre) mit geistiger Behinderung lebt zu Hause bei seinen Eltern und besuchte bislang mehr oder weniger regelmäßig eine Tagesförderstätte. Es gab wiederkehrende Konflikte mit dem Betreuungspersonal, da er häufig zu spät kam oder die Einrichtung vorzeitig verließ und zu Fuß nach Hause lief oder sich in der Stadt umhertrieb.

Die Eltern — der Vater ist gesetzlicher Betreuer — entschieden sich gemeinsam mit ihrem Sohn nach eingehender Beratung durch den Sozialen Dienst des örtlichen Trägers der Sozialhilfe ein Persönliches Budget in Anspruch zu nehmen. Es wurde ein monatlicher Geldbetrag in Höhe von 400 Euro bewilligt und regelmäßig gezahlt. Ein Verwendungsnachweis ist nicht erforderlich.

Auf einer öffentlichen Informationsveranstaltung berichtete der junge Mann selbstbewusst und sichtlich entspannt, was er nun im Unterschied zu früher tagsüber so tue: Mit seinem Vater hat er sich eine Dauerkarte im Fitness-Studio gekauft, das er fast täglich aufsucht. Er berichtet, dass die Mitarbeiter dort viel freundlicher seien. Es gäbe nie Ärger und das Schöne sei, er könne kommen, wann er wolle. Darüber hinaus erfährt man, werde der Restbetrag stetig neu verwendet. Mal zur Anschaffung verschiedener materieller Dinge, mal zur Finanzierung begleitender Hilfen und Urlaubsreisen oder sonstiger alltäglicher Aktivitäten.

Im Ergebnis hat man nicht den Eindruck, dass hier ein junger Mann berichtet, dem das Fehlen einer psychosozialen Förderung durch eine Tagesstätte anzumerken wäre. Im Gegenteil, sein Bericht wirkt für seine Verhältnisse entspannt und souverän.

\section{Beispiel 2: Mallorca statt Wohnheim}

Während derselben Informationsveranstaltung berichtet ein Mann mit seelischer Behinderung (29 Jahre) in Anwesenheit seines gesetzlichen Betreuers über seine bisherigen zahlreichen Aufenthalte in psychiatrischen Kliniken und den sich daran anschließenden Unterbringungen in Wohnheimen. Alle Versuche, in diese Einrichtungen zurechtzukommen, seien gescheitert. Zumeist habe es Auseinandersetzungen wegen der Hausordnungen, der Übernahme von Haus- und Putzdiensten und wegen der 


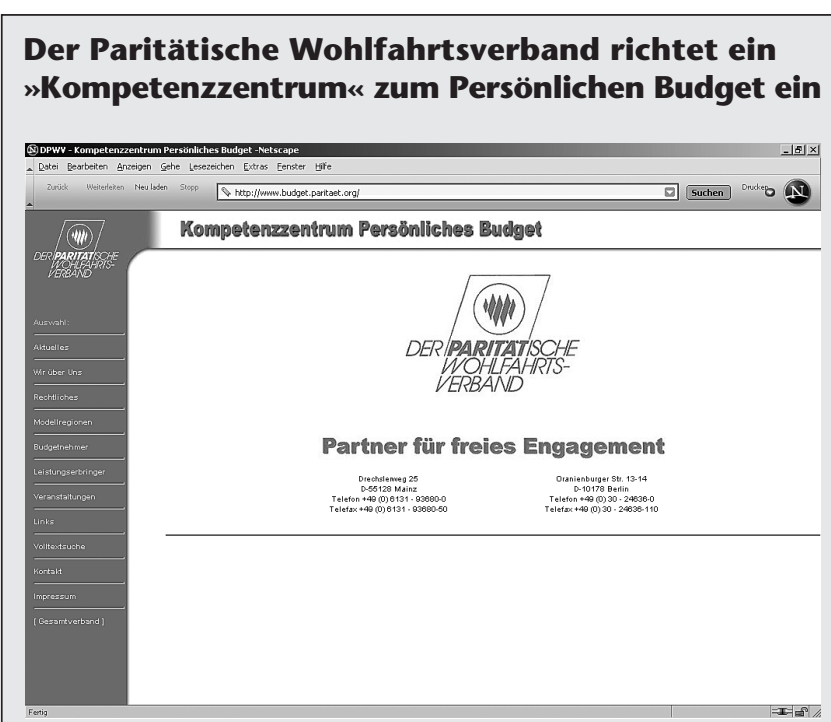

Die neuen Regelungen zum Persönlichen Budget werfen sowohl für die Budgetnehmer als auch für die Leistungsanbieter viele Fragen auf. Behinderte oder pflegebedürftige Menschen sehen sich plötzlich in der Rolle, selbst verantwortlich zu sein für die Organisation ihrer lebensnotwendigen Hilfen. Und den Trägern sozialer Dienste und Einrichtungen stellen sich neue Anforderungen an die Personalplanung, an die Preisgestaltung und an das Marketing. Das PARITÄTISCHE Kompetenzzentrum Persönliches Budget will angesichts dieser sozialpolitischen Umbruchssituation bei der Suche nach fachlich angemessenen und wirtschaftlich tragfähigen Lösungen helfen. In bundesweiten und regionalen Fachtagungen werden in- und ausländische Erfahrungen mit dem Persönlichen Budget dokumentiert und analysiert. Eine per Internet frei zugängliche Website sammelt in einer Datenbank aktuelle Informationen und Grundlagenartikel zum Persönlichen Budget. Das PARITÄTISCHE Kompetenzzentrum Persönliches Budget bemüht sich zudem um Antworten bei Einzelanfragen von Budgetnehmern und Leistungsanbietern.

Das PARITÄTISCHE Kompetenzzentrum Persönliches Budget ist eine gemeinsame Einrichtung des PARITÄTISCHEN Wohlfahrtsverbandes - Gesamtverband e. V. und seiner Landesverbände. Das auf drei Jahre angelegte Modellprojekt wird unterstützt von der Aktion Mensch.

PARITÄTISCHES Kompetenzzentrum Persönliches Budget, Drechslerweg 25, 55128 Mainz, Melanie Fritz 06131 93680-12, E-Mail budget@paritaet.org, Internet http://www.budget.paritaet.org

regelmäßigen Überschreitung von Alkoholverboten gegeben.

Auch er hat sich - nach einschlägiger Beratung durch den Sozialhilfeträger - entschieden, ein Persönliches Budget in Anspruch zu nehmen. Er erhält nun den monatlichen Maximalbetrag von rund 770 Euro.

Im Publikum will man wissen, wozu er das Geld verwende und zur Überraschung der Zuhörer berichtet der Budgetnehmer, dass er das Geld drei Monate gespart habe. Zwischenzeitlich habe er mithilfe und Unterstützung seines gesetzlichen Betreuers in einem Wochenblättchen, das in den örtlichen Haushalten umsonst verteilt werde, ein Inserat aufgegeben: »Bin 29, männlich, seelisch behindert, suche für 8-Woche-Aufenthalt auf den Balearen eine Begleitperson. Zuschriften unter Chiffre erbeten an ....

Er habe vier Bewerbungen erhalten und sich gemeinsam mit seinem gesetzlichen Betreuer für einen jungen Studenten der Sozialpädagogik entschieden. Mit diesem habe er die Regeln und die Bedingungen für eine gemeinsame Reise nach Mallorca ausgehandelt und schriftlich vereinbart. Der Betreuer habe in Palma de Mallorca einen deutschsprachigen Psychiater ausfindig gemacht, mit dem er alle Eventualitäten und Kriseninterventionen im Fall einer psychischen akuten Krise vorbesprochen habe. Dann habe er aus seinem angesparten Budget eine Reise für zwei Personen bei einem Billiganbieter im Reisebüro gebucht und die beiden haben eine gute Zeit auf Mallorca im November und Dezember verbracht.

Auf die erstaunten und teilweise gar empörten Rückfragen der professionellen Leistungsanbieter im Publikum, wo denn hierbei die notwendigen psychosozialen Betreuungsleistungen geblieben wären, antwortet der Budgetnehmer knapp und denkwürdig: »Wissen Sie, das Frühstücksbuffet im Hotel in Palma de Mallorca ist etwas anderes als die Frühstücksgruppe im Wohnheim!«

\section{Beispiel 3: Wohnung statt Wohnheim}

Eine chronisch seelisch behinderte Frau (44 Jahre) lebte nahezu 16 Jahre in psychiatrischen Wohnheimen. Ihr Aussehen und ihre mangelhafte Körperhygiene war stets Anlass zu Auseinadersetzungen und sogenannten »Grenzen setzenden Sanktionen « durch das Betreuungspersonal. Eine dauerhafte depressive Grundstimmung äußerte sich in konsequentem Rückzugsverhalten und steter Ablehnung einer jeden Kooperation.

Der Intervention eines aufgeschlossenen, über das Persönliche Budget gut informierten Mitarbeiters ist es zu verdanken, dass diese Frau heute seit mehr als zwei Jahren selbstständig in einer eigenen Wohnung lebt und in eigener Verantwortung ein Budget von 700 Euro verwaltet, mit dem sie Reinigungspersonal eines hauswirtschaftlichen Dienstes (180 Euro pro Monat), Begleitpersonen für ihre wöchentlichen Einkäufe (60 Euro pro Monat) sowie die 14tägige psychosoziale Beratung (90 Euro pro Monat) bezahlt.

Darüber hinaus hat sie sich in die Arbeits- und Ergotherapiegruppe eines Wohnheims eingekauft. Dort hat sie die Möglichkeit, zweimal in der Woche jeweils zwei Stunden an der Gruppe teilzunehmen und bezahlt dafür monatlich einen Pauschbetrag von 250 Euro an den Wohnheimträger. Darin ist auch das Mittagessen enthalten. Der Restbetrag wird von ihr, wie in den vorangegangenen Beispielen, frei und nach Bedarf verwendet. Häufig dient er zu Finanzierung von Freizeitaktivitäten, zusätzlichen Friseurbesuchen oder einfach zur Verbesserung der ökonomischen Situation.

\section{Beispiel 4: „Betreute Warteliste« statt Unterversorgung}

Ein geistig behinderter Mann mit schweren Verhaltensauffälligkeiten (40 Jahre) lebt zuhause bei seinen Eltern, die beide älter als 70 Jahre sind. Er sollte in ein Wohnheim umziehen, jedoch war die Suche lange erfolglos. Entweder waren die in Frage kommenden Heime nicht bereit, ihn aufgrund seiner Verhaltensauffälligkeiten aufzunehmen oder die zur Verfügung stehenden Plätze waren auf lange Sicht ausgebucht. So steht er heute auf der 
Warteliste eines Heimes auf Platz 14. Was geschieht aber in der Zwischenzeit? Üblicherweise interessiert das die Wohnheimträger weniger, ebenso den Leistungsträger.

Der Rechtsanspruch auf Leistungen zur Teilhabe besteht aber im Einzelfall und zwar genau dann, sobald der Bedarf festgestellt worden ist. Dabei bleibt festzuhalten, der Bedarf auf Leistungen zur Teilhabe artikuliert sich immer in Formulierungen wie »Hilfe zur Selbstversorgung «, »Hilfe zur Tagesgestaltung «, »Hilfe zur Arbeit« u. Ä. Der Rechtsanspruch bezieht sich entsprechend auch nur auf solche Formulierungen. Es gibt keinen Bedarf, der lauten würde: »Ich brauche ein Wohnheim!« Oder: »Ich brauche eines Tagesförderstätte!«

Unter diesen Umständen ist das Konzept der »betreuten Warteliste«, das von uns unter ausdrücklicher Umsetzung des Persönlichen Budgets erfunden worden ist, eine realistische Perspektive, auch in solchen Bedarfssituationen zeitnah tätig werden zu können, in denen Menschen im üblichen Sachleistungssystem nicht sofort auf Hilfe rechnen dürfen.

Der Mensch mit geistiger Behinderung konnte in unserem Beispiel weiter zuhause wohnen bleiben. Er und seine Eltern nutzten die umfassende Budgetberatung, die sie durch einen Wohnheimträger und dessen Case-Management-Beauftragten (nicht identisch mit dem Wohnheimträger, auf dessen Warteliste er sich befand) erhalten haben.

Sie erhielten den maximalen Budgetbetrag zur Sicherung der Teilhabe in Höhe von 770 Euro monatlich durch den Sozialhilfeträger. Zudem wurde die psychiatrische Institutsambulanz (Leistungen nach § 118 SGB V) des Trägers mit der regelmäßigen, aufsuchenden fachärztlichen Behandlung und Betreuung beauftragt. Mit dem Geld aus dem Budget wurden psychosoziale Fachleistungsstunden in Form von Kriseninterventionen bei Bedarf und in der eigenen Familie eingekauft sowie »Babysitterdienste « so bezeichnete die Mutter die Dienste, die durch ambulant-aufsuchendes Betreuungspersonal des beratenden Trägers immer dann erforderlich wurden, wenn die Eltern beide gleichzeitig außer Haus waren und der behinderte Sohn beaufsichtigt werden musste. Verbleibende Restbeträge wurden aufgespart und zur Finanzierung einer begleiteten Einzelreise des Sohnes genutzt.

Als nach acht Monaten der Platz im Wohnheim frei wurde, entschieden sich der behinderte Sohn und seine Eltern gemeinsam, die über das Persönliche Budget gefundene Lösung weiter fortzuführen. Aktuell plant die Familie erstaunlich offen und gemeinsam mit »ihrem « Betreuungspersonal die Zeit nach dem Tod der Eltern.

Insbesondere weiß man nun, dass durch das Budget die unschätzbare Möglichkeit besteht, das Personal weiter zu beschäftigen, zu dem man inzwischen großes Vertrauen gefunden hat. Dabei werden offen die Varianten einer Vorbereitung und Unterbringung in einer Gastfamilie genauso in den Blick genommen wie der Wechsel in eine betreute Wohngemeinschaft, in der allerdings das Personal nicht wie üblich einmal in der Woche vorbeischaut (Schlüssel 1:12), sondern in der dieselben individuell zugeschnittenen komplexen Leistungen erbracht werden, wie jetzt zuhause bei den Eltern.

\section{Fazit}

Das rheinland-pfälzische Persönliche Budget hat seine strukturellen Schwächen: keine Bedarfsorientierung, keine Zielformulierung, kein Verwendungsnachweis, pauschalierte Beträge. Trotzdem zeigt dieses Modellprojekt erstaunliche Ergebnisse in der Sicherung der Teilhabe behinderter Menschen. Die Geldleistung befördert offensichtlich unter diesen Bedingungen günstige Lösungsentwicklungen, die im herkömmlichen Sachleistungsprinzip eher schwierig oder unmöglich sind.

Die Leistungserbringer, die sich an diesen Lösungen beteiligen, verändern ihren Einrichtungsbegriff, ohne allerdings die Einrichtung selbst aufzulösen. Sie bieten eben zusätzlich zum Kerngeschäft ambulant-aufsuchende Leistungen an, für die eine eigenständige Kalkulation und Personalwirtschaft sowie häufig eine andere Leistungsbeschreibung erforderlich ist. Weitaus stärker als bisher sind Leistungen zur Teilhabe im Blick (Stichwort: Lebensqualität und Lebenszufriedenheit).

Nicht sehr gefragt sind die eher »mitarbeiterorientierten « Leistungen des Therapierens, Betreuens, Pflegens, Beratens, Begleitens usw., die von den allermeisten Budgetnehmern als Mittel zum Zweck verstanden werden und für die man sich aufgrund des Wunsch- und Wahlrechts entscheiden kann oder auch nicht. Die Entscheidung trifft der, der das Geld hat. Wer hier als Leistungserbringer weiter »strukturkonservativ« denkt und handelt, der erreicht die Budgetinhaber nicht.

Aufgrund dieser Praxiserfahrungen darf man nun gespannt die Ergebnisse der Modellerprobungen auf der Bundesebene erwarten und es bleibt zu wünschen, dass die verbesserten gesetzlich im SGB IX und der Budgetverordnung sowie im SGB XI verankerten - Strukturbedingungen spätestens dann ab 2008 zu einer bundesweiten Alltagsumsetzung Persönlicher Budgets führen werden.

\section{Anmerkungen}

(1) Damals konnten sich die wenigsten Akteure der Behindertenhilfe vorstellen, dass der Leistungsträger den behinderten Menschen selbst das Geld ausgezahlt. Man hielt das für eine unzulässige Überforderung der Behinderten, die aus reinen Sparmotiven billigend in Kauf genommen würde. Die Empörung wurde zudem noch verstärkt, dass die Geldbeträge, die den Menschen direkt zur Verfügung gestellt wurden, die Vergütungen, die die Leistungserbringer erhielten, weit unterschritten.

(2) § 17 Absatz 3 SGB IX (alte Fassung bis zum 30. Juni 2004): »Die Rehabilitationsträger erproben die Einführung persönlicher Budgets durch Modellvorhaben.«

(3) Persönliche Budgets werden im Rahmen der Leistungen zur Teilhabe nach SGB XII (»Eingliederungshilfe«) an leistungsberechtigte Menschen mit Behinderung alternativ zu einer Sachleistung (»Geld oder Liebe?« — »Wohnheim oder Geld?«) als pauschalierte Geldbeträge in drei Stufen ausgezahlt. Aktuelle Stufungen und Monatsbeträge sind Stufe 1 (200,- Euro), Stufe 2 (400,- Euro) und Stufe 3 (770,- Euro). Korridorbildungen und höhere Beträge in Ausnahmefällen sind möglich.

(4) Vgl. § 17 SGB IX und die Budgetverordnung nach § 21 a SGB IX; siehe auch die Website des PARITÄTISCHEN Kompetenzzentrums Persönliches Budget: http://www.budget.paritaet.org.

(5) Information und Darstellung: http://www.pflegebudget.de.

(6) Information und Darstellung: http://www.integriertesbudget.de.

(7) Die Budgetverordnung schreibt als Bewilligungsvoraussetzung eine schriftliche Zielvereinbarung zwischen Antragsteller und Leistungsträger vor, die insbesondere Zielformulierungen, Regelungen zu Verwendungsnachweisen sowie zur Qualitätssicherung enthalten muss. Die bisherige rheinland-pfälzische Regelung verzichtet völlig auf diese Bedingungen.

(8) Siehe Anmerkung 4. 